\title{
Polysèmes
}

Revue d'études intertextuelles et intermédiales

\section{(Ré)écrire Goldberg : musicalisation, transtylisation et variation chez Gabriel Josipovici}

\section{Marcin Stawiarski}

\section{(2) OpenEdition}

\section{Journals}

\section{Édition électronique}

URL : http://journals.openedition.org/polysemes/681

DOI : $10.4000 /$ polysemes.681

ISSN : 2496-4212

Éditeur

SAIT

Édition imprimée

Date de publication : 1 janvier 2012

Pagination : 227-245

ISSN : 0999-4203

\section{Référence électronique}

Marcin Stawiarski, « (Ré)écrire Goldberg : musicalisation, transtylisation et variation chez Gabriel Josipovici », Polysèmes [En ligne], 12 | 2012, mis en ligne le 01 mars 2015, consulté le 19 avril 2019. URL : http://journals.openedition.org/polysemes/681 ; DOI : 10.4000/polysemes.681

Ce document a été généré automatiquement le 19 avril 2019.

Polysèmes 


\title{
(Ré)écrire Goldberg : musicalisation, transtylisation et variation chez Gabriel Josipovici
}

\author{
Marcin Stawiarski
}

1 Il existe une forme d'ouverture de la littérature à l'art qui retiendra ici plus particulièrement mon attention : sa relation à la musique. La question paraît vaste car la musique peut se manifester dans les textes dans le traitement de la sonorité (la musicalité) ou constituer une source inépuisable de thèmes. Si ces deux aspects n'éveilleront mon attention ici qu'accessoirement, c'est que je souhaite m'attacher à un troisième type de relations qu'entretiennent la littérature et la musique. Il s'agit de la projection de structures musicales dans la fiction, projection par laquelle j'espère pouvoir éclairer la notion de transposition.

2 La structure musicale que je souhaite aborder ici est celle de la variation musicale à travers l'œuvre de l'auteur de langue anglaise, Gabriel Josipovici, qui, ayant déjà à son actif plusieurs romans, nouvelles, pièces de théatre et écrits critiques, publie en 2002 un roman intitulé Goldberg: Variations ${ }^{1}$. Afin d'examiner l'inscription de la forme musicale de la variation au sein de ce roman, nous confronterons en premier lieu trois types de notions qui régissent la présence du musical dans le littéraire: musicalisation, transtylisation et transposition. Puis, c'est à la lumière de ces concepts que je souhaite élucider la dynamique variationnelle chez Gabriel Josipovici, dynamique qui paraît procéder par constante oscillation entre son affirmation et sa négation.

\section{Trois notions voisines : musicalisation, variation, transposition}

3 Écrire la musique, écrire sur la musique, la réécrire ? Comment concevoir l'inscription du musical dans les textes littéraires ? À quel type d'opérations avons-nous affaire au juste? Examinons les trois notions qui vont guider cette analyse. 
4 L'héritage laissé par Aldous Huxley sous forme de ce que l'on peut appeler un roman musicalisé, Point Counter Point, permet de restreindre la problématique. Publié en 1928, il recèle une importante esquisse métatextuelle concernant les rapports entre les deux arts. Le carnet d'un des héros du roman, Philip Quarles, fait l'apologie d'une correspondance musico-littéraire au niveau de la structure romanesque, conférant ainsi au terme de musicalisation $^{2}$ le sens particulier de transposition formelle. C'est à travers les modulations, les transitions ou encore les variations, que l'œuvre littéraire pourra fraterniser avec la musique.

5 La critique musico-littéraire s'est approprié cette dénomination huxleyenne et a pu différencier une approche formelle d'autres manifestations du musical dans le littéraire ( musicalité ou thématique musicale). Se concentrant sur l'ancrage historique de l' intermédialité $e^{3}$, définie comme participation de plus d'un medium à la production du sens dans l'œuvre d'art, Werner Wolf fonde précisément son étude sur la notion de musicalisation de la fiction. En examinant cette pratique, que l'on pourrait situer, en quelque sorte, en marge de l'intertextualité, Werner Wolf en étoffe la portée. La musicalisation $^{4}$ de la fiction apparaît comme une transformation du musical, convié à prendre part à la signifiance littéraire. Le texte tendrait ainsi à ressembler formellement à la musique, à se faire musique et à en donner l'impression. Dans le cas de Goldberg: Variations, c'est par la forme thème et variations que se réalise la musicalisation.

6 À son tour, la notion de variation, au sens général de modification, sous-tend aussi bien l'écriture musicale que littéraire. Selon Calvin S. Brown, les deux arts ont ceci en commun qu'ils structurent leurs matériaux dans un processus d'altération du même ${ }^{5}$. La musique aurait exploité ce phénomène jusqu'à donner naissance à la forme thème et variations tandis qu'en littérature ce genre ne trouve pas de véritable équivalent. Peut-on alors envisager des tentatives d'imitation de cette forme musicale en littérature?

7 Procédé de composition par transformation d'un élément musical, repris sous différents aspects, mais toujours identifiable, la forme variationnelle s'accomplit par l'exposition d'un thème et une suite de variations qui peuvent le modifier au niveau harmonique, mélodique ou encore rythmique. Bien qu'il n'y ait pas de synonyme de ce phénomène dans la production littéraire en tant que genre distinct et codé, nous en trouvons cependant un exemple dans l'œuvre de Raymond Queneau, Exercices de style, phénomène que Gérard Genette a baptisé transtylisation. C'est à la lumière de ce processus, qui met en exergue l'idée du style ${ }^{6}$ comme moyen de la variation, que je souhaite lire la prégnance du musical dans le roman de Josipovici. Recourant à l'exemple des Exercices de style, Gérard Genette ébauche une définition de cette notion qui implique une variabilité stylistique :

Cette œuvre est donc bien, et à juste titre, dans l'esprit de son auteur, une série de variations (stylistiques et autres) sur un même thème (original, mais volontairement neutre ou banal) que chacune de ces variations transforme soit selon un principe mécanique de manipulation de type oulipien, soit en le récrivant dans un style défini [...]. J'appellerai transtylisation ce procédé de la variation stylistique.

Ainsi se trouve défini par Gérard Genette ce cas particulier de la transposition que constitue la réécriture stylistique.

8 Lorsqu'on transpose, on inverse, on permute, on altère, ce qui souligne le caractère dynamique de la transposition. Dans le domaine interartistique, il s'agit du mouvement d'une œuvre vers l'autre, d'un art vers l'autre, et de l'ouverture de l'œuvre à cette 
dynamique plurilatérale. Et si mobilité il y a, c'est que nous sommes aussi dans la sphère du trope.

Le transfert implique la coexistence d'au moins deux pôles différents entre lesquels s'opérerait la transposition. Leur rapprochement relèverait d'un processus analogique qui, comme dans le cas de la métaphore dont l'analogie constitue une sous-espèce ${ }^{8}$, requiert une mise en commun sous forme d'une intersection abstraite. La relation analogique ainsi établie serait une relation triangulaire, dont les deux termes s'aligneraient par le truchement d'une abstraction commune. C'est cette nécessité d'une passerelle abstraite reliant les deux arts qui nous paraît contribuer à la spécificité de la transposition interartistique. Dans La Lecture musico-littéraire, Frédérique Arroyas souligne cet aspect de correspondance entre les arts, en postulant que «la "reconnaissance" de formes musicales dans un texte littéraire demande la création chez les lecteurs de ce qu'on peut appeler une icône, un schème, une figure, c'est-à-dire précisément la constitution d'une instance intermédiaire qui permet le passage du littéraire au musical et vice-versa »'. Ainsi, le transport d'un art à l'autre, à ce niveau particulier de la projection de structures, se réaliserait à la croisée d'abstractions communes. La transposition, apparentée à l'analogie (cette dernière relevant de la métaphore), tendrait vers la création d'une icône entre les arts. Ainsi, je conçois la transposition intersémiotique ${ }^{10}$, terme emprunté à Roman Jakobson, comme la volonté d'inscrire une structure musicale dans une œuvre de fiction par le biais de l'analogie, ce pont abstrait qui constituerait une condition de sa transposabilité.

\section{Les Variations Goldberg : dynamique de la variation / transtylisation}

\section{(Dés)aveu d'une (ré)écriture}

10 Pourquoi lire l'œuvre de Bach en filigrane de Goldberg: Variations de Gabriel Josipovici ? Peut-on faire l'économie de cette filiation et doit-on parler d'une réécriture? Le roman lie certainement le lecteur par un pacte de lecture particulier, qui convoque inéluctablement le musical, mais en même temps semble le tenir à distance.

Au premier abord, c'est dans le paratexte que se signent trois des postulats du contrat de lecture. D'une part, le titre fait appel au musical, à une œuvre célèbre du répertoire baroque, les Variations Goldberg de Bach. C'est une première référence à la musique, mais non pas une identification complète, car le titre, Goldberg: Variations, et non pas Goldberg Variations, s'éloigne de fait du titre que l'on attribue communément à l'œuvre de Bach. Voici donc le premier pacte conclu avec la musique, mais aussi la première rupture.

D'autre part, un symbole musical accompagne le titre. Il s'agit d'une portée à cinq lignes, vide et close de chaque côté par des doubles barres de mesure, celles-ci représentant en musique la fin d'une composition. La présence de ces doubles barres de chaque côté d'une portée paraît symboliser la globalité de l'œuvre de Bach ainsi que sa structure cyclique, la fin équivalant au début. Mais le vide de la portée semble avant tout inviter le lecteur à la remplir, en suggérant ainsi une lecture musicale du roman. Tous les chapitres sont contenus entre ces doubles barres de mesure. Ce deuxième élément ancrant le texte dans le royaume de la musique parait également l'en éloigner ${ }^{11}$. 
13 Le troisième élément qui inscrit le texte dans le musical est constitué par la structure globale du roman, divisé en trente chapitres. Convié par le titre à établir un lien avec l'œuvre de Bach et, par la portée libre, à lire le texte en fonction de la musique, le lecteur peut trouver dans cette division des chapitres la confirmation de la parenté du texte avec les Variations Goldberg, elles-mêmes au nombre de trente. Néanmoins, là aussi, les variations romanesques semblent nier leur lien avec le compositeur. La question est soulignée par Werner Wolf ${ }^{12}$ : le roman de Josipovici paraît avoir abandonné l'Aria, le thème des Variations Goldberg repris à la fin de l'œuvre (le nombre de subdivisions étant alors de trente-deux). L'affinité du romanesque et du musical paraît constamment affirmer puis nier la possibilité de la réécriture.

D'un tout autre ordre est l'alliance du texte avec la musique, s'opérant tantôt par allusion à ce que l'on pourrait appeler le paramusical, tantôt en référence à l'œuvre musicale même et à sa structure. Cette première fait resurgir au sein du roman tout ce qui accompagne l'œuvre de Bach, relevant ainsi de la pure thématique, puisant sa source dans la genèse de l'œuvre ou encore dans la légende qui l'entoure. Le thème du roman a pour origine l'histoire relatée par Nicolas Forkel, premier biographe du compositeur, selon qui les variations dites de Goldberg furent composées à l'instigation du comte Hermann Carl von Keyserling pour chasser ses insomnies. Le protégé de Keyserling, Johann Gottlieb Goldberg, devait exécuter cette œuvre dotée de pouvoirs soporifiques la nuit, depuis une chambre attenante à celle du comte.

Le roman de Gabriel Josipovici met en scène un riche penseur, Westfield, qui, souffrant lui aussi d'épuisantes insomnies, s'avise d'engager un écrivain, nommé Goldberg, pour tenter d'apaiser son affliction en écoutant des histoires que Goldberg devra lui raconter.

16 Ainsi un élément paramusical se trouve-t-il transposé dans le roman où il subit une métamorphose. Le musical est suppléé par le littéraire, marquant une distanciation entre les deux arts et soulevant la question même des relations inter-artistiques. La parenté établie entre la musique et la littérature par ce truchement fait ainsi surgir un désaveu. L'insertion de la genèse des Variations Goldberg affirme ce lien, sa transformation le nie.

17 Finalement, ce sont les références à l'œuvre de Bach, thématique musicale et mentions de toutes sortes, qui tissent un lien indéfectible avec la musique. À titre d'exemple, nous pouvons évoquer le cas de l'expression « cabbages and kings » qui apparaît dans l'avantdernier chapitre. Cette mention énigmatique surprend le protagoniste, (" "Cabbages and kings, Goldberg said." "I beg your pardon? said Westfield." "Cabbages, Goldberg said. And kings." ", 186). Son sens ne peut s'éclairer qu'à la lumière de la trentième variation de Bach, quolibet s'inspirant d'un thème populaire ("cabbage and kings have driven me away $")^{13}$. Le quolibet, pièce musicale constituée de fragments empruntés produisant un effet comique, explique également l'insertion de bribes de chansons populaires dans le chapitre (« My bonny lies over the ocean. My bonny lies over the sea », 188).

Ce réseau de références, d'allusions et de renvois amène le lecteur à penser le texte en fonction de son pendant musical, mais également à repenser ce lien, car le roman de Josipovici s'approprie constamment la musique, tout en faisant mine de s'en déposséder.

\section{La double dynamique textuelle}

19 En dépit de ce paradoxe, le texte parvient néanmoins à consolider son lien avec la musique. Ainsi, il peut être lu comme une variation dont le degré zéro serait constitué 
d'un thème et de ses multiples remaniements à travers divers styles. Cet état idéal mettrait alors en place une double dynamique textuelle.

Tout d'abord, si nous considérons l'œuvre de Gabriel Josipovici comme une variation, il nous semble important d'en envisager le fonctionnement sous couvert de la notion genettienne de transtylisation. Ce procédé de variation littéraire veut que «l'œuvre relève évidemment à la fois de la parodie et du pastiche, puisque chaque variation parodie le thème en pastichant un nouveau style $»^{14}$. C'est précisément cette double dynamique de (ré)écriture qui nous paraît essentielle ici : relation bipolaire, établissant, d'une part, un lien entre chacune des parties (les variations) et le point de départ (le thème), et, d'autre part, entre chaque variation et un style particulier. C'est donc également une dialectique interne / externe : le texte se réécrirait lui-même, tout en en réécrivant d'autres.

Or, cette dynamique transparaît déjà dans la forme strictement musicale du thème et variations. Eu égard à son codage, c'est avant tout dans le domaine musical qu'elle se manifeste le plus distinctement. Le thème apparait comme l'unité de composition. Les différents styles des variations peuvent être plus facilement déterminés (ne serait-ce qu'en fonction de leur entité rythmique). Par conséquent, c'est la variation en tant que forme musicale qui représente le modèle de cette dynamique. Comme le souligne Calvin S. Brown ${ }^{15}$, cette forme ne s'étant pas développée en littérature, elle ne peut prendre vie en tant que forme littéraire que par le truchement du parangon musical. S'ébauche ainsi une véritable projection de structure, se revêtant d'un système réticulé de renvois.

En effet, l'affirmation du lien textuel avec la forme musicale aboutit à forger un réseau dynamique entre le texte et son modèle musical, superposition de deux dynamiques parallèles, impliquant leur projection potentielle. Si la dialectique littéraire de la variation se résout en une parodie du thème et en un pastiche d'un style, si cette même dialectique apparaît dans le domaine musical, on peut supposer, dans le cas de la transposition, un lien du pastiche littéraire avec le style musical. Autrement dit, ce que pasticherait la variation du roman serait également le style musical, consistant déjà en un pastiche, réécrivant ce qui est déjà réécrit. Mais est-il possible qu'un texte imite le style d'un autre art ? Le roman de Gabriel Josipovici paraitt fonder le pastiche de la variation en recourant à différents moyens littéraires (techniques, procédés, styles). Dans Goldberg: Variations, nous pouvons discerner en effet plusieurs situations d'énonciation et divers traits d'écriture. Si l'œuvre devait être considérée alors du point de vue de la transtylisation, elle opérerait la double dynamique mentionnée par Genette. Car le roman côtoie l'épistolaire, le journal intime, le dialogue socratique ou encore des exercices rhétoriques tels que l'ekphrasis. S'accomplirait à travers cette variabilité de procédés ou de traits d'écriture la transposition d'une forme musicale, fondée sur le modèle musical, mais construite à partir de techniques littéraires, mettant en exergue la rivalité entre les deux arts.

Bien qu'elle recoure essentiellement aux procédés littéraires, la variation n'exclut pas le réseau de correspondances pour autant. Au contraire, l'existence de la double dynamique variationnelle qui apparaît en musique et en littérature à la fois, ainsi que la nécessité d'une passerelle entre les deux arts nous semblent caractériser la transposition dans l'œuvre de Josipovici. La variation littéraire s'accomplit dans le modèle musical, mais aussi en dehors de lui. 


\section{Esthétique du revers ; esthétique de la boucle}

Ainsi, nous ne pouvons accorder de pertinence à la notion de transtylisation dans ce texte qu'à la condition d'en envisager l'absence. Nous avons vu que le texte paraît fonctionner par l'affirmation et le déni de sa parenté avec la musique. Mais dénier, c'est déjà partiellement admettre cette présence musicale. Loin de constituer un simple paradoxe, l'inscription de la forme variationnelle au sein du texte et, en apparence, celle de son déni, nous paraissent épouser parfaitement l'esthétique du roman de Gabriel Josipovici : esthétique du revers, esthétique de la boucle.

variations romanesques peuvent se définir dans une certaine mesure comme livre d'illusions. La thématique du trompe-l'œil s'y trouve d'ailleurs abordée dans l'intégralité du onzième chapitre, "Containers", qui peut être considéré, par son caractère sensiblement pictural, comme description d'une œuvre d'art, une ekphrasis :

A shelf in a shallow recess, above which is a cupboard with two small doors, one of which is partially open, but not enough to allow one to see inside, the other firmly shut. Each door is fastened to the frame by two elaborate metal hinges, which extend to within an inch or two of the further edge and which, at the point of juncture, take up about a quarter of the vertical length of the door. (Goldberg: Variations, 67)

C'est dans le quatorzième chapitre que l'on en trouve un important écho, comme une continuation de cette description, et où la figure de l'ekphrasis trouve son fondement du fait même qu'il s'agisse d'une œuvre d'art :

Utterly different from anything else in the museum, it was at once self-contained and utterly mysterious. Divided horizontally into two parts, it showed a shallow cupboard, the top half consisting of two doors, one of which was partly open in trompe-l'œil, the lower half consisting of a shelf on which stood a number of containers, while more - bottles, flasks and the like - hung from nails set in the wall. (101)

L'inscription d'un trompe-l'œil dans une dynamique d'échos renforce le jeu de miroirs dans le texte. Le caractère énigmatique du onzième chapitre se donne à lire rétrospectivement, grâce au chapitre quatorze, comme la description d'un cabinet de curiosités. Aussi le texte crée-t-il une esthétique particulière de miroirs, d'échos, de renversements, à laquelle participent de nombreuses mises en abyme comme celle du seizième chapitre, "The Challenge", portant sur une improvisation en forme de variations, le modèle même du roman.

Trompe-l'œil, écho, miroir, le texte adopte une esthétique qui tend à montrer le revers de l'objet qui deviendrait objet à son tour, esthétique qui s'empare également du cycle ou de la boucle. C'est le premier chapitre du roman qui parvient à tracer une boucle étrange (" a strange loop ») ${ }^{16}$, où la face devient le revers, le contenu le contenant et vice versa. Arrivé chez son employeur (Westfield) à qui Goldberg doit faire la lecture la nuit pour l'endormir, le héros va devoir écrire lui-même des histoires et être confronté à un manque absolu d'inspiration. Il décide alors de composer justement sur le thème de l'impossibilité d'écrire et du manque d'inspiration: «If that happens to be a labyrinth from which there seems to be no exit, that will become my theme. If it is the frustrating search for a subject which refuses to emerge, then that will be my theme» (10). Aussi le texte dénude-t-il le revers de sa textualité, et il ne tardera pas à mimer ce renversement.

Polysèmes, 12 | 2015 
29 En effet, ce premier chapitre se présente comme un récit de Goldberg (sa première journée chez Westfield), mais dès le début, la présence d'un destinataire confère au texte un trait épistolaire ("[he] asked me about you and the children», 2). Or, à la fin du chapitre, lors d'une conversation avec Westfield, Goldberg décide d'écrire son histoire sous forme de lettre à son épouse («I would write the composition for which you asked in the form of a letter to my wife. Not any letter, you understand, sir, but a letter telling her of my visit here and of the circumstances in which I now find myself », 11). Lequel des récits se trouve affecté par ce trait épistolaire ? Celui que l'on est en train de lire, celui que Goldberg va raconter à Westfield ou encore celui que le héros raconte véritablement à son épouse ? C'est justement l'illusion que le texte instaure, tendant à brouiller ici les récits. Si le récit épistolaire, que l'on croit relaté par Goldberg à son épouse, soudain devient le récit que Goldberg va raconter à Westfield, la fin précédant ainsi le début, c'est que le texte opère un glissement de l'hypothétique à un simple dialogue. En effet, Goldberg écrit à son épouse :

After supper I sat at the desk and wrote. Alas, I no longer even tried to fulfil my obligations to my employer, but instead I wrote to you. I knew of course what he would say. He would call me in and sit me again by his bed, as he had on the previous night.

- Well - Well, my friend, he would say, why do you sit there in silence when you are acquainted with my wishes? (9. C'est moi qui souligne)

Suit un dialogue que l'on croit au premier abord imaginaire, car relevant de l'hypothétique, qui apparaît dans les verbes introducteurs qui soutiennent le dialogue, mais celui-ci disparaît rapidement, et la conversation se tourne vers la lettre même qu'il écrit à son épouse. Ainsi, l'illusion consiste à faire passer une hypothèse pour ce qui aurait réellement fait partie des événements. Le contenu de la lettre devient son contenant: trompe-l'œil parfait.

31 Or les notions de cycle, de mise en abyme ou de boucle ne sont pas sans lien avec l'œuvre de Bach. La mise en abyme transparaît dans les Variations Goldberg au niveau harmonique et numérologique. Les trente-deux parties de l'œuvre (Aria, trente variations, Aria da capo) trouvent leur pendant dans la basse elle-même en trente-deux mesures. Si l'œuvre peut être divisée en deux sous-parties de quinze variations et une Aria chacune, chacune des variations se laisse diviser en deux versants (l'harmonie, avec le passage de la tonique à la dominante et vice versa, établissant en général un chiasme, $\mathrm{T}-\mathrm{D}-\mathrm{D}-\mathrm{T}$, donc un miroir). Finalement, le retour cyclique est symbolisé non seulement par le retour à la tonique de la plupart des variations, mais il se reflète avant tout dans la reprise da capo de l'Aria. Ces quelques éléments démontrent la parenté du roman de Josipovici avec l'œuvre de Bach en termes d'esthétique du cycle, du revers ou du miroir. Ils paraissent indiquer que la musique constitue l'une des sources de cette esthétique pour l'œuvre romanesque. La musicalisation, ou plus précisément la transtylisation, participerait à l'esthétique romanesque de l'illusion.

C'est précisément dans cette esthétique que s'inscrit la problématique de la variation littéraire. Afin d'opérer à la manière de la musique, le degré fondamental de la forme variationnelle doit affirmer un thème qui subirait une suite de transformations. Nous avons constaté une dynamique de l'affirmation et du désaveu de la présence du musical dans le texte de Gabriel Josipovici. Ce qui, de fait, constitue la pierre d'achoppement la plus importante $d u$ processus variationnel, c'est le manque du thème. Comment concevoir le texte en fonction de son négatif musical si se manifestent des incompatibilités entre les deux ? Les trente parties à l'œuvre (au lieu de trente-deux dans 
les Variations Goldberg) signifient-elles que le thème (Aria) en est supprimé ? Nous pensons que cette incommensurabilité peut être justement mesurée à l'aune de la distanciation entre le littéraire et le musical dans le roman, constituant son désaveu. Il est certes essentiel d'envisager un degré fondamental, degré zéro de la transposition, et d'admettre ainsi l'existence potentielle d'une diversité, et par là même, d'une étanchéité stylistiques entre les variations, les différents chapitres du roman. Or, à la place de cette entité abstraite, nous remarquons une perméabilité, plutôt qu'une étanchéité, entre les différentes variations.

Thématiquement, les échos, les parallélismes et les miroirs instaurent un réseau de passerelles entre les variations. Plutôt que de parodier un thème, une variation en parodierait une autre. Stylistiquement, certains traits caractéristiques d'un chapitre se refléteraient dans un autre. C'est, par exemple, le cas de l'épistolaire qui parait s'immiscer dans un chapitre à la manière d'un journal intime (quinzième chapitre, «Mrs. Goldberg»), instaurant un destinataire de journal, donc une ambiguïté entre l'autodestination et l'allodestination:

When you are absent I take to my notebook. It is the only way I know of being with you. It has always been like that. Martha will always be scribbling, Papa used to say. I cannot remember when I discovered the comfort that writing brings. Nobody will read it, not even myself. Yet writing things down, bending over the white page, dipping the pen in the ink, pausing, looking up, starting again - all that brings release and appeasement, such as merely closing the eyes and imagining never does. (103)

Cet extrait, imitatif du journal intime, fait également écho au premier chapitre en ce sens qu'il peut être reçu comme une réponse à l'écrit épistolaire de Goldberg. Par conséquent, Goldberg, destinateur du premier chapitre, se trouve destinataire de ce journal qui acquiert ainsi une dimension épistolaire ( Are you reading at the moment to Mr. Westfield? », 108 ; « How I love you, Mr. Goldberg! », 110). Il y aurait là un processus antonyme de la variation, une anti-variation. Si l'on considère l'« uniformité », définie justement comme "absence de variation", comme un antonyme de la variation, nous retrouvons l'idée du même, donc le thème. Nous revoilà dans l'esthétique du revers et du cycle. C'est la recherche de la variation qui constituerait le thème du roman. C'est donc aussi un roman sur la quête de l'inspiration romanesque.

L'examen de l'œuvre de Gabriel Josipovici au prisme de la musique m'incline à conclure que le roman suscite ou ravive un différend entre les deux arts. Concevant la transposition à l'échelle d'un transfert d'une forme d'art vers une autre par l'intermédiaire d'un rapport analogique, j'ai essayé de démontrer qu'une telle dynamique dans le cas de Goldberg: Variations procède par son affirmation et sa négation. Ce rapport de forces participe à l'esthétique du roman de Josipovici qui, tout en se construisant par rapport à l'œuvre de Bach, s'en distancie. En conséquence, penser la notion de transtylisation comme pendant, voire équivalent, de la forme musicale du thème et variations parait impossible à son degré fondamental. Le roman semble admettre cette possibilité, tout en la déjouant, et c'est précisément ce paradoxe de musicalisation qui paraît caractériser cette réécriture. 


\section{BIBLIOGRAPHIE}

Aristote. Poétique. Paris : Belles Lettres, 1965.

Arroyas, Frédérique. La Lecture musico-littéraire. Montréal : Presses de l'Université de Montréal, 2001.

Brown, Calvin S. Music and Literature: A Comparison of the Arts. Hanover and London: University Press of New England, 1987.

Cupers, Jean-Louis \& Ulrich Weisstein. Word and Music Studies: Musico-Poetics in Perspective. Calvin S. Brown in Memoriam. Amsterdam: Rodopi, 2000.

Genette, Gérard. Palimpsestes. La Littérature au second degré. Paris : Seuil, 1982.

Hofstadter, Douglas R. Gödel, Escher, Bach: An Eternal Golden Braid. New York: Basic Books, 1979.

Huxley, Aldous. Point Counter Point. London: Dalkey Archive Press, 1996.

Jakobson, Roman. Essais de linguistique générale. Paris : Éditions de Minuit, 1963.

Josipovici, Gabriel. Goldberg: Variations. Manchester: Carcanet, 2002.

Queneau, Raymond. Exercices de style. Paris : Gallimard, 1947.

Williams, Peter. Bach: The Goldberg Variations. Cambridge: CUP, 2001.

Wolf, Werner. The Musicalization of Fiction: A Study in the Theory and History of Intermediality. Amsterdam: Rodopi, 1999.

Wolf, Werner. "The Role of Music in Gabriel Josipovici's Goldberg: Variations". Style 37.3 (2003): 294-317.

\section{NOTES}

1. Gabriel Josipovici, Goldberg: Variations, Manchester: Carcanet, 2002.

2. Aldous Huxley, Point Counter Point, London: Dalkey Archive Press, 1996, 294 : "The musicalization of fiction. Not in the symbolist way, by subordinating sense to sound. (Pleuvent les bleus baisers des astres taciturnes. Mere glossolalia). But on a large scale, in the construction [...]. A theme is stated, then developed, pushed out of shape, imperceptibly deformed, until, though still recognizably the same, it has become quite different ".

3. Werner Wolf, The Musicalization of Fiction: A Study in the Theory and History of Intermediality, Amsterdam: Rodopi, 1999, $1:$ " The participation of more than one medium of expression in the signification of a human artifact ».

4. Ibid., 52 : «The musicalization of fiction may be defined as a special case of covert musicoliterary intermediality to be found in (parts of) novels or short stories. It consists in a (in most cases intentional) shaping of the discours (affecting, e.g., the linguistic material, the formal arrangement of structure of the narrative, and the imagery used) and sometimes also of the histoire (the content structure of the narrative) so that verifiable or at least convincingly identifiable "iconic" similarities or analogies to (a work of) music or to effects produced by it emerge in the fictional text. As a result, the reader has the impression that music is involved in 
the signifying process of the narrative not only as a general signified or a specific - real or imaginary - referent but also that the presence of music can indirectly be experienced while reading ".

5. Calvin S. Brown, Music and Literature: A Comparison of the Arts, UP of New England, 1987, 103 : « Repetition and variation can be seen in the smallest real structural units of both literature and music ».

6. Par commodité nous utilisons ici la notion de style. Il conviendrait de la nuancer à la lumière de ce que l'on appelle, en musicologie, une variation de caractère, appellation que l'on utilise pour étudier l'œuvre de Bach.

7. Gérard Genette, Palimpsestes. La Littérature au second degré, Paris : Seuil, 1982, 165.

8. Cf. Aristote, Poétique, Paris : Belles Lettres, 1965, 60 : «La métaphore est le transport à une chose d'un nom qui en désigne une autre, transport ou du genre à l'espèce, ou de l'espèce au genre, ou de l'espèce à l'espèce ou d'après le rapport d'analogie ».

9. Frédérique Arroyas, La Lecture musico-littéraire, Montréal : Les Presses de l'Université de Montréal, 2001, 90.

10. Roman Jakobson, Essais de linguistique générale, Paris : Éditions de Minuit, 1963, 86 : «Seule est possible la transposition créatrice : transposition à l'intérieur d'une langue - d'une forme poétique à une autre -, transposition d'une langue à une autre, ou, finalement transposition intersémiotique - d'un système de signes à un autre, par exemple de l'art du langage à la musique, à la danse, au cinéma ou à la peinture ».

11. Les doubles barres de mesure n'apparaissent en général qu'en fin de composition. L'on pourrait pourtant envisager de débuter une œuvre par sa fin lorsque son commencement équivaut à sa fin. Un exemple notable est fourni par le type spécifique du canon qu'est le canon à l'écrevisse, un vrai palindrome musical que l'on rencontre dans L'Offrande musicale de Bach. Par ailleurs, le canon est au cœur des Variations Goldberg.

12. Werner Wolf, "The Role of Music in Gabriel Josipovici's Goldberg: Variations", Style 37.3 (2003): 294-317.

13. Pour la référence à l'allusion chez Bach («Ich bin so lang nicht bei dir gewest Kraut une Rüben haben mich vertrieben »), voir Peter Williams, Bach: the Goldberg Variations, Cambridge: CUP, 2001, 88-92. Tout en affirmant la pratique citationnelle, cette allusion à l'emprunt dans l'œuvre de Bach paraît également épouser le thème de l'itération et de l'éloignement dans le roman de Josipovici.

14. Genette, op. cit., 165.

15. Voir l'article "Theme and Variations as a Literary Form", in Jean-Louis Cupers \& Ulrich Weisstein, Word and Music Studies: Musico-Poetics in Perspective, Calvin S. Brown in Memoriam. Amsterdam: Rodopi, 2000, ainsi que les chapitres "Repetition and Variation" et "Theme and Variations" in Calvin S. Brown, Music and Literature, op. cit. Brown se réfère à un certain nombre de problèmes que pose la transposition de la variation en littérature, parmi lesquels figurent celui de la longueur et celui du thème.

16. Comme l'envisage Douglas R. Hofstadter dans Gödel, Escher, Bach: An Eternal Golden Braid, New York: Basic Books, 1979, 717-719. 
INDEX

oeuvrecitee Goldberg: Variations 